\title{
Charm Production in Neutral Current Neutrino-Nucleus Scattering within the Color Dipole Approach
}

\author{
M. B. Gay Ducati ${ }^{a}$, M. M. Machado ${ }^{a}$, and M. V. T. Machado \\ ${ }^{a}$ High Energy Physics Phenomenology Group, GFPAE, IF-UFRGS \\ Caixa Postal 15051, CEP 91501-970, Porto Alegre, RS, Brazil and \\ ${ }^{b}$ Universidade Federal do Pampa. Centro de Ciências Exatas e Tecnológicas, \\ Campus de Bagé, Rua Carlos Barbosa. CEP 96400-970. Bagé, RS, Brazil
}

(Received on 20 March, 2008)

\begin{abstract}
The rate for inclusive charm production in neutral current neutrino-nucleus interactions is calculated. The interaction of high energy neutrinos on hadron targets is an outstanding probe to test Quantum Chromodynamics (QCD) and shed light in the understanding of the parton properties of hadron structure. We have considered the QCD color dipole picture for such an interaction and have in addition used parton saturation models. In particular, the dipole cross section is taken from recent phenomenology on deep inelastic scattering (DIS) at DESY-HERA. The theoretical predictions are compared to available theoretical predictions and experimental results from $\mathrm{NuTeV}$ detector at Fermilab. Moreover, we compute the relative rate of the associated cross section in deep inelastic neutrino-nucleus interactions, $\sigma(c \bar{c} \mathrm{v}) / \sigma_{t o t}^{N C}$, from CHORUS Collaboration. As a byproduct, a QCD analysis of the $F_{2}, F_{L}$ and $F_{3}$ structure functions for the neutral current case also is presented.
\end{abstract}

Keywords: Charm production; Dipole picture; Neutrino-nucleus interaction

\section{INTRODUCTION}

Neutrino interactions are classified as charged current $(\mathrm{CC})$ or neutral current (NC) processes, depending if a virtual $W^{ \pm}$ or $Z^{0}$ boson gauge is exchanged. In the first case, a charged lepton is emitted, while in the second case the neutrino conserves its nature. The neutral current process played an important role in the establishment of the Standard Model of electroweak interactions. Experimental studies with NC neutrino interactions is a demanding task due to the considerable difficulties of data extraction on reactions with cross sections smaller than $\mathrm{CC}$ process, where the event identification has to rely on the detection of one or more hadrons. Furthermore, NC neutrino-proton interactions are also important to give an answer for a challenging question: what is the charmquark contribution to the proton structure function [1]. Observations of open charm production in neutral current deep inelastic neutrino scattering are seen in the NuTeV detector at Fermilab, where the production model and the observed level of charm production is used to determine the effective charm mass [1]. The main evidence for neutral current charm production in $v_{\mu} N$ or $\bar{v}_{\mu} N$ scattering is an unconfirmed observation of $J / \psi$ production [2,3]. Relative rate of the associated charm cross-section in deep inelastic $v$-interactions has been measured as $\sigma(c \bar{c} v) / \sigma_{\mathrm{NC}}^{\mathrm{DIS}}=\left[3.62_{-2.42}^{+2.95}(\right.$ stat $) \pm 0.54($ syst $\left.)\right] \times$ $10^{-3}$ [4] at low energy. It is expected from QCD that the charm contribution is increasingly large at higher energies. The rate for charmed content in ultra high energy (cosmic ray) neutrino interactions is quite important in the correct simulation of particle showers to be measured in neutrino telescopes.

In this contribution, we calculate the total cross section and the associated proton structure functions using the color dipole formalism [5] for the neutral current neutrino-proton scattering. Such an approach is quite successful in describing the high energy behavior of the nucleon structure functions in lepton-nucleon (nucleus) interactions and its presentation is somewhat intuitive. It has been corroborated by sev- eral phenomenological studies on small- $x$ structure functions and gives good results in the charged current neutrino interactions [6]. Here, we consider a recent parameterization for the dipole-nucleon cross section, which it was shown [6] to give a good description of data in the small- $x$ region. We organize the present contribution as follows: In Section 2, the structure functions $F_{2}, F_{L}$ and $F_{3}$ are investigated using the color dipole picture and taking into account a phenomenological parton saturation model [7], as well as the total neutrinonucleon cross section. The explicit expressions for NC structure functions and total NC cross section are summarized in that section. In the last section, we present our numerical results with emphasis on the cross section of charm production $\sigma_{\text {charm }}$ and the rate $\sigma_{\text {charm }} / \sigma_{\text {tot }}$. The results are compared to the available theoretical predictions and experimental data.

\section{HIGH ENERGY NEUTRINO-NUCLEON SCATTERING}

Considering the neutral current reaction of the type $\mathrm{v}(k) N(p) \rightarrow \mathrm{v}\left(k^{\prime}\right)+X\left(p^{\prime}\right)$ with the following notation for four-momenta $k_{\alpha}=\left(E_{v}, \vec{k}\right), k_{\alpha}^{\prime}=\left(E_{v}^{\prime}, \vec{k}^{\prime}\right), p_{\alpha}=(E, \vec{p})$ and $p_{\alpha}^{\prime}=\left(E^{\prime}, \vec{p}^{\prime}\right)$, the differential cross section describing it can be expressed as [8]

$$
\begin{gathered}
\frac{\partial^{2} \sigma_{v, \bar{v}}^{N C}}{\partial x \partial y}=\frac{G_{F}^{2} M E_{v}}{\pi}\left(\frac{M_{Z}^{2}}{Q^{2}+M_{Z}^{2}}\right)^{2}\left[\frac{y^{2}}{2} F_{L}^{N C}\left(x, Q^{2}\right)\right. \\
\left.-\frac{1+(1-y)^{2}}{2} F_{2}^{N C}\left(x, Q^{2}\right) \pm y\left(1-\frac{y}{2}\right) F_{3}^{N C}\left(x, Q^{2}\right)\right],
\end{gathered}
$$

where $G_{F}$ is the Fermi constant, $M_{Z}$ denotes the mass of neutral gauge boson. Furthermore, $Q^{2}=-q^{2}$ is the boson virtuality, $x=\frac{Q^{2}}{2 p q}$ and $y=\frac{p q}{M E_{\mathrm{v}}}$ (inelasticity variable), with $M$ is the nucleon mass. The neutrino energy is denoted by $E_{\mathrm{v}}$ and $p, q$ are the four momenta of the nucleon and of lepton exchange boson, respectively. $F_{L}, F_{2}$ and $F_{3}$ stand for the corresponding 


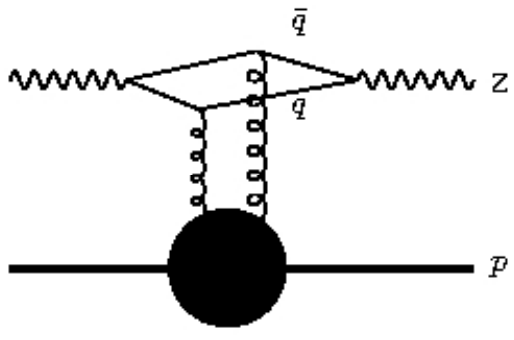

FIG. 1: Schematic view of neutral current neutrino-proton interaction in the color dipole formalism.

structure functions. We take the usual relation [9]:

$$
F_{L}\left(x, Q^{2}\right)=\left(1+4 \frac{x^{2} m_{p}^{2}}{Q^{2}}\right) F_{2}\left(x, Q^{2}\right)-2 x F_{1}\left(x, Q^{2}\right)
$$

The QCD parton model gives the structure functions $F_{2,3}\left(x, Q^{2}\right)$ expressed in terms of the quark and antiquark distributions in nucleon [9]. Therefore, considering an isoscalar target $N=\frac{n+p}{2}$ for the NC process one has:

$$
\begin{aligned}
& F_{2}^{N C}\left(x, Q^{2}\right)=\frac{Q_{W}}{4}\left\{x\left[u_{v}\left(x, Q^{2}\right)+d_{v}\left(x, Q^{2}\right)\right]\right. \\
& \left.+2 x\left[\bar{u}\left(x, Q^{2}\right)+\bar{d}\left(x, Q^{2}\right)+s\left(x, Q^{2}\right)+c\left(x, Q^{2}\right)\right]\right\}
\end{aligned}
$$

and

$$
F_{3}^{N C}\left(x, Q^{2}\right)=\frac{Q_{W}}{4}\left[u_{v}\left(x, Q^{2}\right)+d_{v}\left(x, Q^{2}\right)\right]
$$

where we used the notation $Q_{W}=L_{u}^{2}+L_{d}^{2}+R_{u}^{2}+R_{d}^{2}$ and the chiral coupling can be expressed in terms of the Weinberg angle $\theta_{W}[8]$ :

$$
\begin{array}{cl}
L_{u}=1-\frac{4}{3} \sin ^{2} \theta_{W}, & L_{d}=-1+\frac{2}{3} \sin ^{2} \theta_{W}, \\
R_{u}=-\frac{4}{3} \sin ^{2} \theta_{W}, & R_{d}=\frac{2}{3} \sin ^{2} \theta_{W} .
\end{array}
$$

The structure function $F_{2}$ depends on the valence, $q_{v}\left(x, Q^{2}\right)$, and sea quark content, $q\left(x, Q^{2}\right)$, of the isoscalar nucleon. At high energies, the sea contribution dominates due to the abundant gluonic emissions in that kinematic region. The $x F_{3}$ structure function is basically given by the valence content of nucleon, which is a vanishing contribution at very high energies. In this region, a successful framework to describe the deep inelastic scattering is the color dipole formalism [5], which gives a very good description of the small- $x$ structure functions. The physical picture of DIS at low- $x$ can be understood in a sequence of three factorized processes: (a) the virtual boson fluctuates into a color neutral $Z^{0} \rightarrow q \bar{q}$ dipoles, (b) the interaction of this dipole with the nucleon and, finally, (c) the final state is produced. This is depicted in Fig. (1).
The interaction is modeled considering a dipole-target cross section, whereas the boson splitting in a color dipole quarkantiquark is given by the corresponding wavefunction. In the color dipole formalism, the structure functions read as [7, 9]:

$$
F_{T, L}^{N C}\left(x, Q^{2}\right)=\frac{Q^{2}}{4 \pi^{2}} \int d^{2} \vec{r} \int_{0}^{1} d z\left|\Psi_{T, L}^{Z^{0} \rightarrow q \bar{q}}\right|^{2} \sigma_{d i p}(x, \vec{r}),
$$

where $\vec{r}$ denotes the transverse size of the color dipole, $z$ the longitudinal momentum fraction carried by the quark and $\Psi_{T, L}^{Z^{0}}$ are the wavefunctions for transverse or longitudinal polarization of boson, which are expressed by [9]

$$
\begin{aligned}
\left|\bar{\Psi}_{T}^{Z^{0}}\right|^{2} & =\frac{3 Q_{W}}{2 \pi^{2}}\left\{\left[z^{2}+(1-z)^{2}\right] \bar{Q}^{2} K_{1}^{2}(\bar{Q} r)+m_{f}^{2} K_{0}^{2}(\bar{Q} r)\right\} \\
\left|\bar{\Psi}_{L}^{Z^{0}}\right|^{2} & =\frac{6 Q_{W}}{\pi^{2}}\left[z^{2}(1-z)^{2}\right] Q^{2} K_{0}^{2}(\bar{Q} r)
\end{aligned}
$$

where $\bar{Q}^{2}=z(1-z) Q^{2}+m_{f}^{2}, K_{0,1}(u)$ are the modified Bessel functions and $m_{f}$ is the effective quark mass. Here, we have used, $m_{u, d}=0.14 \mathrm{GeV}, m_{s}=0.3 \mathrm{GeV}$ and $m_{c}=1.5 \mathrm{GeV}$. The remaining heavy quark contributions have been disregarded in the present calculation. The phenomenological parameterization from Golec-Biernat-Wusthoff (GBW model) is able to describe experimental data on inclusive and diffractive deep inelastic $e p$ scattering at small- $x$. Therefore, we have used it in our numerical calculations. The parametrisation for the GBW dipole cross section takes the eikonal-like form,

$$
\sigma_{d i p}(\bar{x}, \vec{r})=\sigma_{0}\left[1-\exp \left(-\frac{Q_{s}^{2}(x) \vec{r}^{2}}{4}\right)\right], x
$$

where the saturation scale $Q_{s}^{2}(x)=\left(\frac{x_{0}}{\bar{x}}\right)^{\lambda}$ defines the onset of the saturation effects. The parameters $\left(\sigma_{0}, \lambda\right.$ and $\left.x_{0}\right)$ were obtained from a fit to the HERA data [7]. The variable $\bar{x}=x\left(1+4 m_{f}^{2} / Q^{2}\right)$ gives a suitable transition to the photoproduction region $\left(Q^{2} \rightarrow 0\right)$. The uncertainties coming from different parameterizations for the dipole cross section is expected to be small. As the color dipole model is suitable in the region below $x=0.01$ and the large- $x$ limit still needs a consistent treatment, we supplement the dipole cross section with a threshold factor $(1-x)^{n}$, where $n=5$ (7) for number of flavours $n=3(4)$. When considering a nuclear target we have replaced the dipole-nucleon cross section by the dipolenucleus cross section using the Glauber-Gribov formalism. Namely, we consider the relation [10]:

$$
\sigma_{A}(x, \vec{r} ; A)=2 \int d^{2} b\left[1-\exp \left(-\frac{1}{2} T_{A}(b) \sigma_{d i p}(x, \vec{r})\right)\right],
$$

where $b$ is the impact parameter of the dipole-nucleus scattering and $T_{A}(b)$ is the nuclear profile function [11].

Let us now compute the structure functions for the $\mathrm{NC}$ neutrino-nucleon scattering. In Fig. 2, we present the structure $F_{2}$ as a function of $x$ (left panel) and boson virtuality (right panel). In each case, we have selected representative points for fixed $x, Q^{2}$. As it is expected from a QCD point 

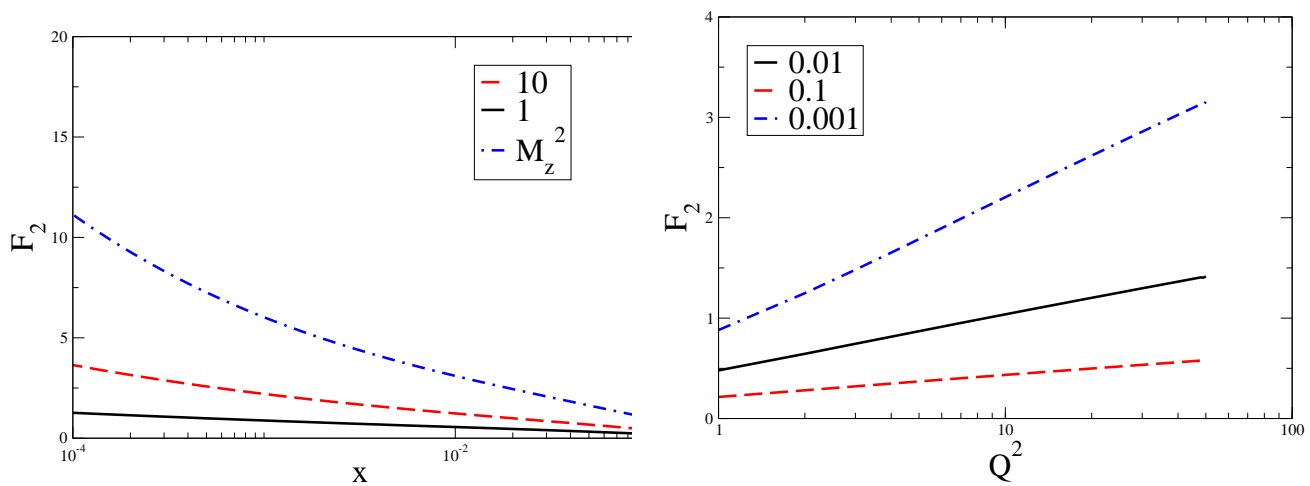

FIG. 2: The $F_{2}^{N C}\left(x, Q^{2}\right)$ structure function for distinct values of $Q^{2}=1,10, M_{Z}^{2} \mathrm{GeV}^{2}$ as a function of $x$ (left panel). The same function for fixed $x=0.1,0.01,0.001$ as a function of the boson virtuality (right panel).

of view, the structure function presents a strong increasing towards small- $x$ at fixed $Q^{2}$. Accordingly, the scaling violations are observed for the virtuality dependence at fixed- $x$. In Ref. [6] we have verified that different implementations for the dipole cross section produce similar numerical results. Then, we will use the GBW model as a baseline for further analysis. We call attention that for the isoscalar target, the $x F_{3}$ structure function is vanishing, as can be seen in Eq. (4). In Fig. 3 we present the corresponding structure function for the charm case, which show similar behavior as the total structure function. However, for virtualities below charm threshold, $\mu^{2}=4 m_{c}^{2}$, the contribution is vanishing (see left panel for $Q^{2}=1 \mathrm{GeV}^{2}$ and right panel for $Q^{2} \ll \mu^{2}$ ). The present calculation should be timely for open charmed mesons in electroweak interactions.

The dipole picture is particularly suitable for a qualitative analysis as the physical interpretation is clear in this representation. For instance, taking Eq. (6) and the explicit expression for the overlap of wavefunctions, Eqs. (7) it is well known that the the scattering process receives contributions from the symmetric dipole configurations, where $\langle z\rangle=1 / 2$, and from the aligned jet (asymmetric) dipole configurations $(z \rightarrow 0$ or $z \rightarrow 1$ ). If the characteristic size of the $q \bar{q}$-pair is much smaller than the mean distance between partons, $R_{0}(x)=1 / Q_{s}$, it can be shown that qualitatively the structure function $F_{2}$ behaves as,

$$
F_{2}^{N C}\left(x, Q^{2}\right) \propto \sigma_{0} Q_{s}^{2}(x)\left[2+\log \left(\frac{Q^{2}}{Q_{s}^{2}}\right)\right],
$$

where the main contribution $\sigma_{0} Q_{s}^{2}$ comes from the regions $r<$ $2 / Q$ and $r>2 / Q_{s}$. The logarithm enhancement comes from the region $2 / Q<r<2 / Q_{s}$. The same qualitative study can be done for the charm quark contribution. In particular, the scale $\mu^{2}=4 m_{c}^{2}$ sets distinct behavior for the charmed structure function,

$$
F_{2}^{c \bar{c}}\left(x, Q^{2}\right) \propto\left\{\begin{array}{ll}
\sigma_{0} Q_{s}^{2}(x)\left[1+\log \left(\frac{Q^{2}}{4 m_{c}^{2}}\right)\right], & : \quad Q^{2} \gg 4 m_{c}^{2} \\
\frac{\sigma_{0} Q_{s}^{2}(x)}{4 m_{c}^{2}} Q^{2} & : \quad Q^{2} \ll 4 m_{c}^{2}
\end{array},\right.
$$

To find the size of charm production in a NC neutrinonucleon interaction, we take the ratio of the cross section for charm production, $\sigma_{\text {charm }}$, by the total $\mathrm{NC}$ cross section, $\sigma_{\text {tot }}^{N C}$,

$$
R_{c \bar{c}}=\frac{\sigma_{\mathrm{charm}}}{\sigma_{\text {tot }}^{N C}}
$$

where, in order to compute them we use the following limits of integration in integrating Eq. (1): $0 \leq x, y \leq 1$ [8]. Accordingly, we expect that the amount of charm is increasingly important as neutrino energy growths. This is particularly important for the ultra high energy (cosmic rays) neutrino interactions.

\subsection{Results and Conclusion}

Let us compute the size of charm contribution to the total NC neutrino-nucleon cross section. In Tab. (I) we have the results for the charm production cross section $\sigma_{\text {charm }}$, total cross section $\sigma_{\text {total }}$ and the rate $\sigma_{\text {charm }} / \sigma_{\text {tot }}$ in neutrino-proton interactions for values of neutrino energy 27 and $154 \mathrm{GeV}$ and an extrapolation to higher energies. It should be stressed that for this neutrino energies we are in the limit of validity of color dipole approach. The Bjorken variable in this case can be approximated by $x \simeq Q^{2} /\left(2 M E_{v}\right)$. Then, we are integrating over $x_{\min } \leq x \leq 1$, where $x_{\min } \equiv Q_{\min }^{2} /\left(2 M E_{\mathrm{v}}\right)=10^{-3}\left(10^{-5}\right)$ for $E_{\mathrm{v}}=27,(154) \mathrm{GeV}$. We have considered $Q_{\min }^{2}=0.01 \mathrm{GeV}^{2}$. This values can be compared to the experimental values extracted by the either low energy measurements of CHORUS Collaboration (27 GeV) [4] and NuTeV Collaboration (154 $\mathrm{GeV}$ ) [3]. Our prediction is consistent with the expectation of an increasingly large charm contribution to the total cross section. Namely, we have at low energies $R_{c \bar{c}} \simeq 0.01$, going up to $R_{c \bar{c}} \simeq 0.3$ at very high energies.

The results presented here consider only the sea quark contribution to the structure functions, and in this way we have only charm in the final state (no inclusion of valence quarks contribution). Despite the limitations of the current approach for the low energy neutrino energy, our estimates are comparable to NC neutrino-nuclei data for charm production. Our calculation is also consistent with other approaches in the literature at small- $x$ (high energies). For instance, it is compatible to the results in Refs. [9, 12], where distinct treatments for 

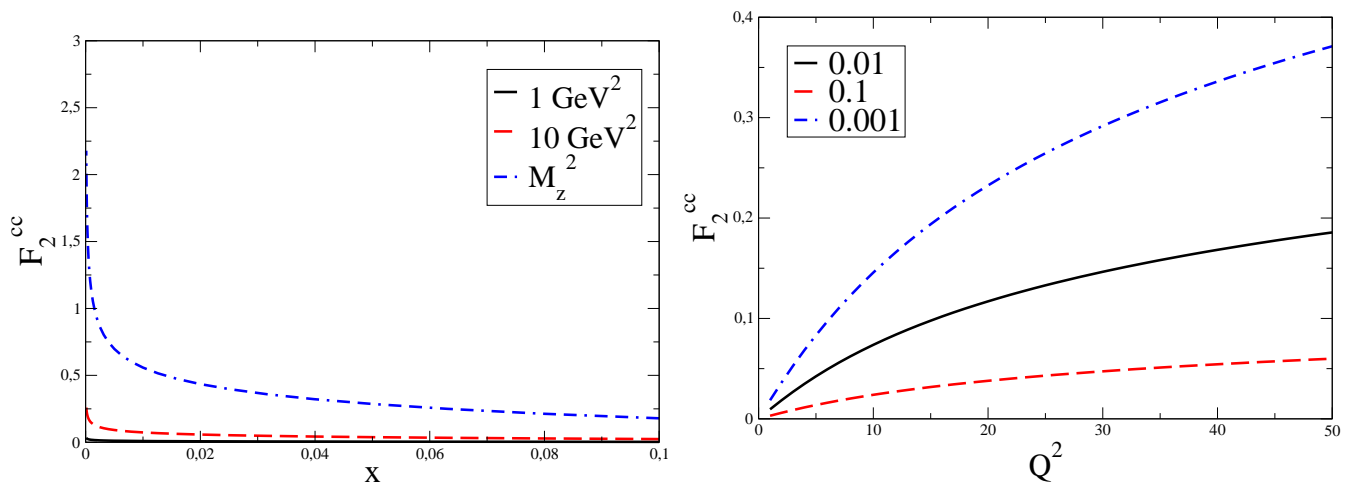

FIG. 3: The NC charm structure function, $F_{2}^{c \bar{c}}\left(x, Q^{2}\right)$. Same notation as previous figure.

TABLE I: Results for the charm production cross section $\sigma_{\text {charm }}$, total NC neutrino-nucleon cross section $\sigma_{\text {tot }}^{N C}$ and the ratio $\sigma_{\text {charm }} / \sigma_{t o t}$ for different values of the neutrino energy.

\begin{tabular}{|l|r|r|r|}
\hline \hline Energy $(\mathrm{GeV})$ & $\sigma_{\text {tot }}^{N C}\left(\mathrm{~cm}^{2}\right)$ & $\sigma_{\text {charm }}\left(\mathrm{cm}^{2}\right)$ & $R_{c \bar{c}}$ \\
\hline $27(\mathrm{CHORUS})$ & $2.0 \times 10^{-38}$ & $5,4 \times 10^{-40}$ & 0.027 \\
\hline $154(\mathrm{NuTeV})$ & $1.4 \times 10^{-37}$ & $1,9 \times 10^{-38}$ & 0.135 \\
\hline 1000 & $4.6 \times 10^{-36}$ & $7.1 \times 10^{-37}$ & 0.154 \\
\hline 10000 & $1.6 \times 10^{-35}$ & $3.0 \times 10^{-36}$ & 0.188 \\
\hline 100000 & $1.5 \times 10^{-34}$ & $3.3 \times 10^{-35}$ & 0.225 \\
\hline \hline
\end{tabular}

the small- $x$ region are taken into account. It would be worth to include QCD evolution in the calculation of total neutrino cross section and valence quarks should be added. Therefore, we believe that the dipole formalism gives a robust theoretical approach to describe the structures functions in neutrino interactions in the low- $x$ region.
[1] A. Alton et al., Int. J. Mod. Phys. A 16S1B, 764 (2001).

[2] H. Abramowicz et al., [CDHS Collaboration], Phys. Lett. B 109, 1 (1982).

[3] T. Adams et al. [NuTeV Collaboration], Phys. Rev. D 61, (2000).

[4] CHORUS Collaboration, Eur. Phys. J. C 52, 543 (2007).

[5] A. H. Mueller, Nucl. Phys. B 335 (1990) 115;

N. N. Nikolaev, B. G. Zakharov, Z. Phys. C 49, 607 (1991).

[6] M. M. Machado, M. B. Gay Ducati, and M. V. T. Machado, Phys. Lett. B 644, 340 (2007).

[7] K. Golec-Biernat, M. Wusthoff, Phys. Rev. D 59, 014017
(1999);

K. Golec-Biernat, M. Wusthoff, Phys. Rev. D 60, 11402 (1999).

[8] R. Gandhi, C. Quigg, M. H. Reno, and I. Sarcevic, Astropart. Phys. 5, 81 (1996); Phys. Rev. D 58, 093009 (1998).

[9] K. Kutak and J. Kwieciński, Eur. Phys. J. C 29, 521 (2003).

[10] N. Armesto, Eur. Phys. J. C 26, 35 (2002).

[11] C. W. De Jager, H. De Vries, and C. De Vries, At. Data Nucl. Data Tables 14, 479 (1974).

[12] J. Kwienciski, A. D. Martin, and A. M. Stasto, Phys. Rev. D 59, 093002 (1999). 